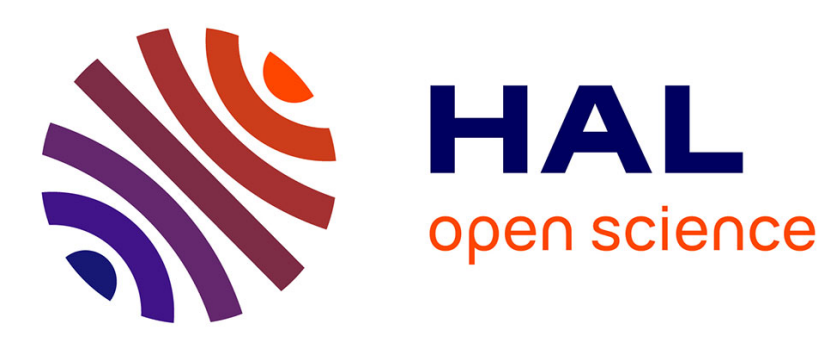

\title{
Realization and simulation of wall shear stress integrated sensors
}

Delphine Meunier, Sedat F. Tardu, Dimitrios Tsamados, Jumana Boussey-Said

\section{To cite this version:}

Delphine Meunier, Sedat F. Tardu, Dimitrios Tsamados, Jumana Boussey-Said. Realization and simulation of wall shear stress integrated sensors. Microelectronics Journal, 2003, 34, 10.1016/S00262692(03)00203-9 . hal-00261997

\section{HAL Id: hal-00261997 \\ https://hal.science/hal-00261997}

Submitted on 4 Feb 2020

HAL is a multi-disciplinary open access archive for the deposit and dissemination of scientific research documents, whether they are published or not. The documents may come from teaching and research institutions in France or abroad, or from public or private research centers.
L'archive ouverte pluridisciplinaire HAL, est destinée au dépôt et à la diffusion de documents scientifiques de niveau recherche, publiés ou non, émanant des établissements d'enseignement et de recherche français ou étrangers, des laboratoires publics ou privés. 


\title{
Realization and simulation of wall shear stress integrated sensors
}

\author{
Delphine Meunier ${ }^{\mathrm{a}}$, Sedat Tardu ${ }^{\mathrm{b}, *}$, Dimitrios Tsamados $^{\mathrm{a}}$, Jumana Boussey $^{\mathrm{a}}$ \\ anstitut de Microélectronique, Electromagnétisme et Photonique, IMEP, ENSERG, 23 rue des Martyrs, 38016 Grenoble Cédex, France \\ ${ }^{\mathrm{b}}$ Laboratoire des Ecoulements Ge'ophysiques et Industriels, LEGI, INPG-UJF-CNRS, B.P. 53 X, 38402 Grenoble Ce'dex, France
}

\begin{abstract}
In this paper we present the impact of hot wire geometry and fluid characteristics on the efficiency of a hot wire to be used as a wall shear stress sensor. Finite differences and finite elements modelling based simulators were used in order to evaluate the thermal performances of hot wire wall shear stress sensor. Several structures were explored including simple conductor or suspended above a cavity (free and sealed with an oxide membrane). It is found out that wire length, wire section and the dimensions of the micro-cavity lying below the hot wire are of fundamental importance on the thermal exchanges occurring between the hot wire and the fluid.
\end{abstract}

Keywords: MEMS; Wall hot film and pressure micro-sensors; Micro-wire over cavity; Numerical simulation; Heat transfer

\section{Introduction}

The wall shear stress in aerodynamics is of capital importance in particular in wall turbulent flows. It gives not only a clear diagnosis of the near wall structures, but also is directly related to the active control of drag. The direct measurement of the wall shear stress fluctuations is quite difficult (Tardu [1]). Its indirect determination is based on hot-film, hot-wire anemometry. A small element is flush mounted at the wall (Fig. 1) and is maintained at a constant temperature. The heat flux from the hot film to the fluid is then proportional to the $1 / 3$ power of the shear in ideal situations for which the parasitic effects such as the conduction to the substrate, or the axial conduction are negligible. Such effects may seriously deteriorate the frequency response of the sensor.

The aim of the present study is to develop simple wall shear stress sensors using the MEMS technology. Some wall shear stress sensors with different configurations exist in the literature, but their efficiency has not been clearly shown up to now (Liu [2]). One of the main reasons of possible deficiency of such micro-sensors may be the importance of the heat conduction from the hot-film

\footnotetext{
* Corresponding author. Tel.: + 33-4-76827030; fax: + 33-4-76825271.

E-mail address: sedat.tardu@hmg.inpg.fr (S. Tardu).
}

elements to the oxide diaphragm on which they are deposited. The aim of the present investigation is to overcome this effect by considering a hot wire into a micro-cavity flush mounted at the wall. It may be considered that in this case, the ratio of heat conductivities of the working fluid and the substrate is one. This small conductivity ratio enhances well the dynamic response of the micro-sensor. We deal in this study, with the realization and the related numerical simulations of the fluid-flow-heat transfer process over a wall sensor with this particular geometry. We first present 2D finite difference, direct and complete simulations of the dynamic response of a hot film under different fluid/substrate conditions. We then detail the realization process of two wall shear stress sensor prototypes, realised with MEMS technology. Conventional Finite Volume Tools (FVM) tools are subsequently used to determine their performance.

\section{2D Numerical simulations of a hot film sensor}

We first briefly outline some 2D complete numerical simulations in order to point at the effect of the conduction into the substrate on the frequency response. A numerical simulation of the heat transfer from a flush mounted wall 


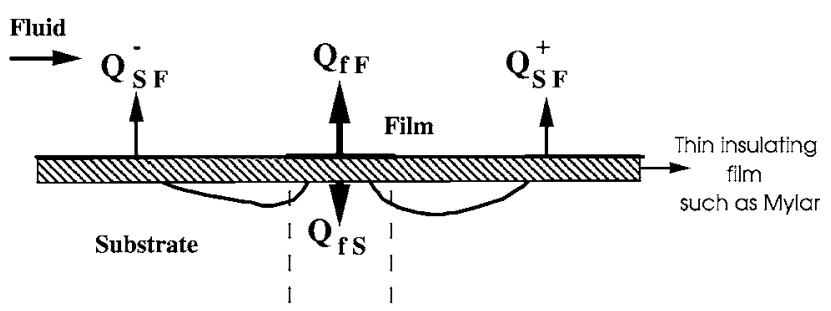

Fig. 1. Heat transfer process over a wall hot film gauge.

hot-film (WHF) (Fig. 1) used for measurement of the wall shear stress $\tau$ is carried out to investigate:

(i) its response in reversing flow

(ii) the effects of heat conduction to the substrate.

The full problem with the heat transfer equations in the fluid (F) and in the solid (S) coupled at the F-S interface (except on the WHF where the temperature $\theta_{\mathrm{f}}$ is imposed; $\mathrm{f}$, for film) is solved for an oscillating flow wherein the wall shear stress varies periodically in time with $\tau=$ $\bar{\tau}(1+a \cos \omega t), \bar{\tau}$ standing for the time mean average of the wall shear stress. The solution depends upon the following parameters: $\omega^{*}=\omega l_{\mathrm{f}}^{2} / \alpha_{\mathrm{F}}, \sigma=\operatorname{Pr}\left(l_{\mathrm{f}}^{+}\right)^{2}, \alpha_{\mathrm{F}} / \alpha_{\mathrm{S}}$ and $k_{\mathrm{F}} / k_{\mathrm{S}}$ $\left(l_{\mathrm{f}}=\right.$ streamwise length of film, Pr: Prandtl number, $\alpha, k=$ heat diffusivity, conductivity, + is the quantity nondimensionalized by the shear velocity $u_{\tau}$ and the viscosity $\nu$ ).

The heat transfer law generally used for wall shear stress measurements $\left(N u=\right.$ const. $\sigma^{1 / 3}$ where $N u$ stands for the global Nusselt number over the film) is based on the simplest solution which assumes $\partial \theta / \partial t=\partial^{2} \theta / \partial x^{2}=0$ in the fluid (quasi-steadiness and no longitudinal diffusion) and $\alpha_{\mathrm{S}}=0$. More complete solutions have been considered in the past, for instance with $\partial \theta / \partial t \neq 0$, or with $\alpha_{\mathrm{S}} \neq 0$ (in the 1D case with a substrate thickness $=l_{\mathrm{f}}$ ). A qualitative analysis (Tardu [1]) has implicitly taken into account the conductivity ratio $k_{\mathrm{F}} / k_{\mathrm{S}}$ in an attempt to explain the differences measured on the intensity of the turbulent shear stress fluctuations $\overline{\tau^{\prime}}$ in air and in water. Yet this alone does not explain some observations of Dengel [3] which show that the measured $\overline{\tau^{\prime} \tau^{\prime}}$ is closer to the true value on a well conducting substrate than on an insulator. The full simulation introduces the additional parameter of the diffusivity ratio $\alpha_{\mathrm{F}} / \alpha_{\mathrm{S}}$ which points to the importance of how fast the heat is transferred to the fluid after conduction through the solid in addition to how much heat is transferred this way as compared to the direct transfer on the film.

\subsection{Numerical method}

The variables in the temperature equation are nondimensionalized with the following quantities: length, $l_{\mathrm{f}}$; time, $l_{\mathrm{f}}^{2} / \alpha_{\mathrm{F}}$; temperature difference, $\Theta_{\mathrm{f}}-\Theta_{\infty}$; velocity, shear velocity $u_{\tau}=\sqrt{\bar{\tau} / \rho}$ where $\rho$ is the density of the fluid and $\bar{\tau}$ is the time mean wall shear stress.
The non-dimensional equations in the fluid $(F)$, in the solid $(\mathrm{S})$, and at the fluid-solid interface $(\mathrm{F}-\mathrm{S})$ are, respectively:

$$
\begin{aligned}
& \mathrm{F}: \partial \theta / \partial t+u \partial \theta / \partial x=\partial^{2} \theta / \partial x^{2}+\partial^{2} \theta / \partial y^{2} \\
& \mathrm{~S}:\left(\alpha_{\mathrm{F}} / \alpha_{\mathrm{S}}\right) \partial \theta / \partial t=\partial^{2} \theta / \partial x^{2}+\partial^{2} \theta / \partial y^{2} \\
& \mathrm{~F}-\mathrm{S}:\left(k_{\mathrm{F}} / k_{\mathrm{S}}\right) \partial \theta_{\mathrm{F}} / \partial y=\partial \theta_{\mathrm{S}} / \partial y
\end{aligned}
$$

where $u=\sigma y(1+a \sin \omega t)$ is the oscillating velocity. In the present computations, the amplitude is throughout $a=0.5$.

The boundary conditions are $\partial \theta / \partial x=0$ and $\partial \theta / \partial y=0$ for $x, y \rightarrow \infty$. The size of the computational domain is in the $x$ direction: $10-50 l_{\mathrm{f}}$; in the $y$ direction: $2-4 l_{\mathrm{f}}$ in the fluid and $10-20 l_{\mathrm{f}}$ in the solid substrate. Upstream weighted differencing and Alternating Direction Implicit method (ADI), combined with stretched coordinates-near the trailing and leading edge of the WHF as well as near the wall-have been used.

The code was tested on the exact 1D time dependent three layer case $\left(\mathrm{F}-\mathrm{S}-\mathrm{F}\right.$, with solid thickness $=l_{\mathrm{f}}$ and with the same flow on both sides of the solid slab.

\subsection{Response in unsteady flow. Effect of the conduction into the substrate}

The unsteady computations were started from the time mean steady field and were carried on until the difference in temperature at any point was less than $0.01 \%$ between two successive cycles. The computational domain must be adapted to each flow and fluid-substrate combination. Its streamwise extent was progressively increased until the wall temperature on the upstream boundary was less than 0.1 .

The anemometer measures the total heat transfer from the film $(Q)$ to the fluid $Q_{\mathrm{fF}}$ and to the solid $\left(Q_{\mathrm{fS}}\right)$ i.e. one has $Q=Q_{\mathrm{fF}}+Q_{\mathrm{fS}}$ (Fig. 1). In the unsteady regime, there cannot be an instantaneous balance between $Q_{\mathrm{fs}}$ and the sum of transfer upstream and downstream of the film $Q_{\mathrm{SF}}^{-}+$ $Q_{\mathrm{SF}}^{+}$, as in the steady regime, because the heat flux variations imposed at the solid surface by the velocity changes are filtered to variable degrees by the substrate, depending upon the distance to the film and the frequency.

Some values of the frequency response of the wall hot film are plotted in Fig. 2. The limiting value of the attenuation is $1 / 5$ when the mean flow parameter is $\sigma=30$. This value is in reasonable agreement with the reduced turbulent intensity of the wall shear-stress measured by some authors in air flows. It is seen that the amplitude is already reduced by a factor one half when $\omega^{*}=3 \times 10^{-4}$. The frequency response for the water/glass combination is reasonably acceptable, while the thin insulating mylar film, improves somewhat the response compared with the air/glass case, but this improvement is far from being totally satisfactory.

Owing to the finite transit time over the film $l_{\mathrm{f}} / u_{\tau}$, the thermal boundary layer itself has a finite frequency response. But this frequency limit $\left(\omega^{*} \cong 1\right)$ is much higher than the limit imposed by the transfer via the substrate. 


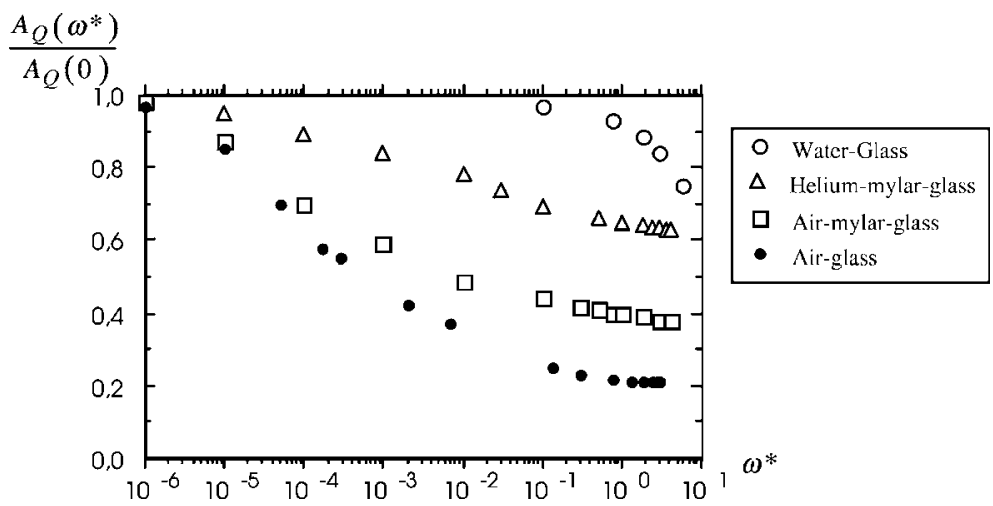

Fig. 2. Frequency response of different fluid/substrate configurations. The conductivity ratios are glass/water: 1.3, mylar/air: 4.9, glass/air: 25.3, glass/helium: 5.2, mylar/helium: 1 .

The numerical investigation of the heat transfer of wall mounted hot films shows that heat transferred to the fluid via the substrate has important effects. Thus, for high values of the conductivity ratios $k_{\mathrm{S}} / k_{\mathrm{F}}$, the transfer from the film to the substrate may be considerably larger than the direct transfer to the fluid. For the air/glass combination, the transfer to the fluid through the substrate is about 10 times larger than the direct transfer. Because of the hot wake, the downstream transfer from the substrate to the fluid is roughly two times smaller than the one upstream.

The importance of the conduction into the substrate increases with decreasing velocity. A thin layer of material attenuates this effect with low conductivity inserted between the film and substrate.

The frequency response of the total heat transfer from the film, as measured by an anemometer set, is dominated by the filtering effect of the transient heat transfer via the substrate when $k_{\mathrm{F}} \ll k_{\mathrm{S}}$ and $\alpha_{\mathrm{F}} \gg \alpha_{\mathrm{S}}$. For the air/glass combination, the response is attenuated as soon as $\omega^{*}>$ $10^{-6}$. This frequency limit is much lower than that of the response of the thermal layer in the fluid itself.

The satisfactory frequency response of the case water/glass $\left(k_{\mathrm{S}} / k_{\mathrm{F}}=1.3\right)$ does not mean that there is no parasitic heat conduction to the substrate at all. On the contrary, the ratio $Q_{\mathrm{fS}} / Q_{\mathrm{fF}}$ varies between 1 and $2 / 3$ as it is seen in Fig. 3a. Yet, this ratio reaches values as large as 10 in the case air/ glass $k_{\mathrm{S}} / k_{\mathrm{F}}=25.3$ (Fig. 3b). The heat loss ratios of roughly $Q_{\mathrm{fS}} / Q_{\mathrm{fF}} \approx 1$ do not affect considerably the frequency response at least up to $\omega^{*}=1$.

The ratio $k_{\mathrm{S}} / k_{\mathrm{F}}$ should obviously be roughly one to have an acceptable dynamic behaviour of the wall film gage. This fact has to be taken into account in MEMS applications. The polysilicon wire is deposed on nitride or silicon oxide diaphragms in some designs (Liu [2]). The silicon oxide has a $14 \mathrm{~W} / \mathrm{m} / \mathrm{K}$ thermal conductivity, which is very low compared to the silicon $(160 \mathrm{~W} / \mathrm{m} / \mathrm{K})$ and polysilicon $(50 \mathrm{~W} / \mathrm{m} / \mathrm{K})$. Yet, the ratio of conductivities for silicon oxide/air case reaches huge values of 500 and severe heat conduction effects to the diaphragm are expected even when the later is of $1 \mu \mathrm{m}$ or so thick. The simplest solution to avoid the effects of the heat conduction into the substrate for air is to consider a wall wire into a micro-cavity. The air into the cavity plays at least qualitatively the role of substrate and $k_{\mathrm{S}} / k_{\mathrm{F}}=1$ by definition. A first version of such a sensor gave very satisfactory results concerning the statistics of the wall shear stress turbulent fluctuations up to fourth moments (Tardu [4]). We therefore decided to realise these hot wire gauges in their MEMS version.

\section{Realization of wall shear stress sensors in MEMS technology}

We have realised two models of sensor:

- a simple shear stress sensor requiring only three mask levels where the hot wire (in polycrystalline silicon)
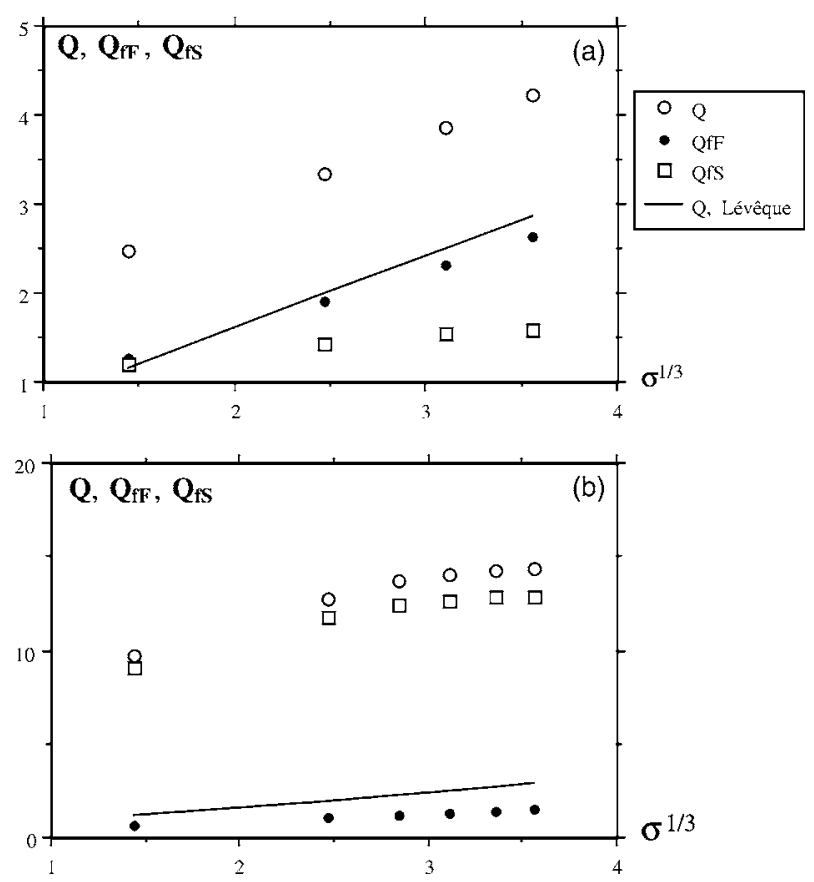

Fig. 3. Non-dimensional heat transfer rates from the film to the fluid and substrate $\left(Q_{\mathrm{fF}}, Q_{\mathrm{fS}}\right)$ and the total heat transfer rate $Q$ versus the nondimensional shear parameter $\sigma$ for (a) water/glass, $k_{\mathrm{S}} / k_{\mathrm{F}}=1.3$, (b) air/glass, $k_{\mathrm{S}} / k_{\mathrm{F}}=25.3$. 


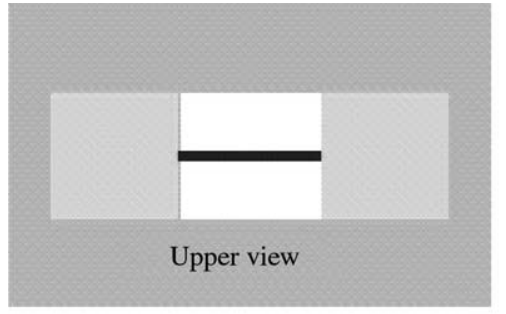

Cavity

Aluminium Pad

Poly silicon Hot wire

Silicon nitride

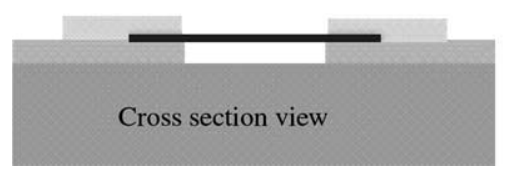

Silicon substrate

Fig. 4. Technological realization of the hot-wire probe on a micro-cavity.

is thermally isolated from the substrate by an air cavity.

- another shear stress sensor requiring eight mask levels based on SOI wafer bonding.

Surface micro-machining was chosen thanks to its capability of providing thin surface cavities and other suspended elements. This is definitely an advantage in this application as the device should not disturb the near wall flow by its presence.

\subsection{Realization of a simple shear stress sensor}

Fig. 4 shows the technological process adopted for the realization of the sensors. Plasma Enhanced Chemical Vapour Deposition (PECVD) silicon nitride was first deposited on $4^{\prime \prime}$ wafers to a thickness of $0.3 \mu \mathrm{m}$. Then the nitride was patterned, and wet thermal oxidation of silicon made through the nitride window. Oxidation time was adjusted to achieve flat surface. A $0.5 \mu \mathrm{m}$ depth silica cavity was obtained. Polycrystalline silicon was deposited (LPCVD—thickness $1 \mu \mathrm{m})$ and doped by boron ion implantation.

After the thermal annealing, polycrystalline silicon was patterned into a $50 \times 2 \mu \mathrm{m}^{2}$ wire. Electrical contacts were finally taken with aluminium. Releasing of the wire was performed by wet-etching of the silica cavity after dicing the sensors. Fig. 5a shows the released $2 \times 50 \times 0.3 \mu \mathrm{m}^{3}$ wire over the $0.5 \mu \mathrm{m}$ depth cavity. Fig. 5b illustrates a double hot-wall wire that will allow the experimental simultaneous determination of the two components of fluctuating shear stresses at the wall and facilitate the active control of the near wall turbulence [1]. The cut-off frequency of this sensor is measured to be $2 \mathrm{kHz}$, and is estimated to be 10 times larger when it will be integrated in a constant temperature anemometer.

\subsection{Realization of a shear stress sensor based on SOI technology}

We have developed another technology based on SOI wafer in order to determine two other important
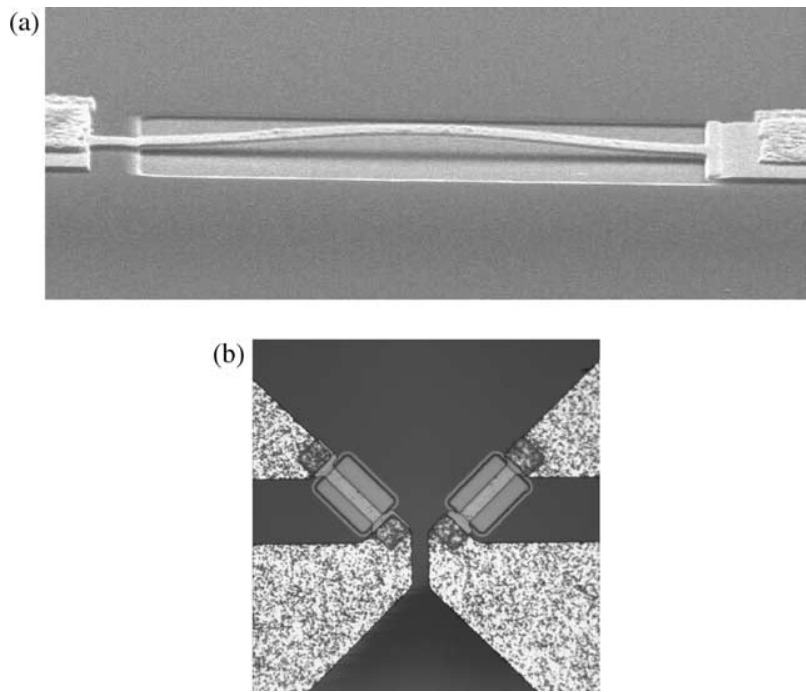

Fig. 5. The wall shear stress probe in its MEMS configuration. (a) Single sensor on a micro-cavity $(0.5 \mu \mathrm{m}$ depth). The dimensions of the sensor are $50 \times 2 \times 0.3 \mu \mathrm{m}^{3}$. (b) Double sensor to simultaneously measure the two components of the wall vorticity.

parameters (pressure and temperature) for the flow fluctuations detection. The detailed process flow chart is shown in Fig. 6. It is based on the use of two SOI wafers. The first one (base wafer) is a UNIBOND wafer with thick enough SOI layer in which the pressure sensor cavities are elaborated by wet chemical silicon etching (Fig. 6a) which ensures a high degree of reproducibility and a perfect depth uniformity. The second SOI wafer is characterized by a thinner SOI film and is simply patterned with shallow cavities (Fig. 6b). After appropriate surface cleaning treatment, the two wafers are assembled by a direct fusion bonding procedure, and the whole silicon substrate of the second wafer is removed by CMP. The as-obtained structure (Fig. 6c) is then ready for the subsequent fabrication steps including gauges implantation, polysilicon deposition and patterning for hot wire anemometer and final metallization.

\section{Numerical modelling}

\subsection{Simulations of a shear stress sensor made of a polysilicon hot wire suspended on an air cavity}

The wall wire on the micro-cavity behaves essentially as a hot-wire, for which special care has to be paid because the effects of axial (i.e. spanwise) conduction. To be short, it can be shown that the ratio of the spanwise length to the square root of the wire cross-section has to be larger than at least approximately 40 in order to have a relatively uniform temperature distribution (Tardu [1]). It may be seen that the sensor we realised fulfils this condition. 


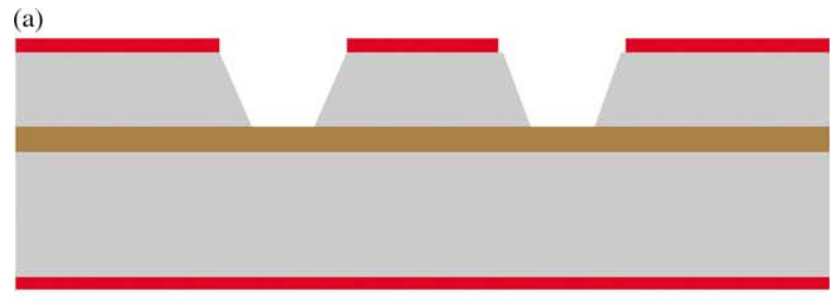

(b)

(c)
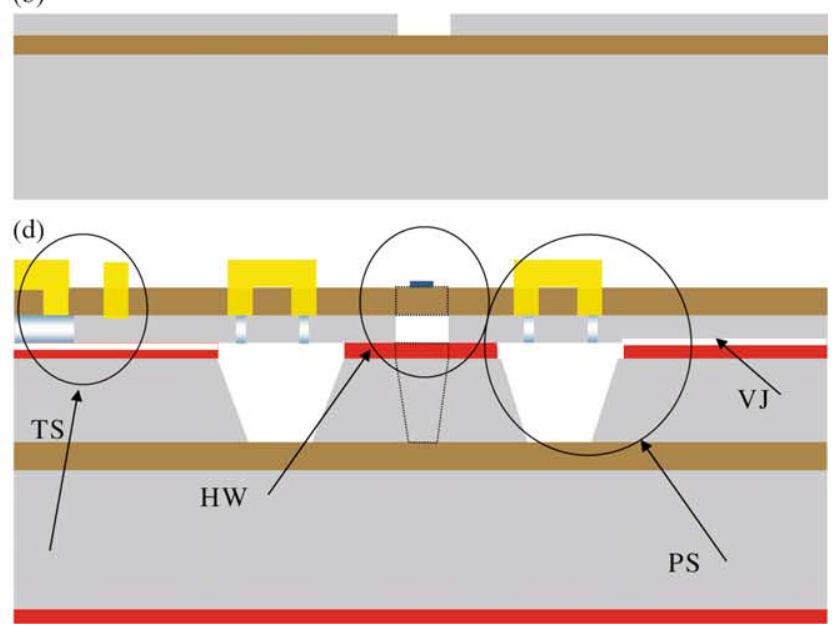

Fig. 6. Chart of the SOI based process for multisensors fabrication. (a) Pressure sensor cavity elaboration in the 'base' BESOI wafer by anisotropic etching of silicon. (b) Patterning within the 'active' SOI wafer of the cavity underneath the hot wire anemometer. (c) A new substrate is obtained by bonding together the above-processed wafers and thinning down the upper active layer. (d) Final structure showing the three types of sensors. Few deeper cavities underneath the hot wire (dotted lines) are realized in step 1. TS: Temperature sensor, HW: Hot wire, PS: Pressure sensor, VJ: Vent hole.

We present now 3D numerical simulations of the wall wire over the micro-cavity (Fig. 5) performed by a commercially available FVM code (Flotherm). The simulated structure (Fig. 7) is composed of four layers, namely:

- a $40 \mu \mathrm{m}$-thick mono-silicon substrate

- a $2 \mu \mathrm{m}$-thick silicon dioxide film

- a $1 \mu \mathrm{m}$-thick silicon mono-silicon film

- a $1 \mu \mathrm{m}$-thick silicon dioxide film

The two last films (mono-silicon and silicon dioxide) will be etched in order to realise a $2 \mu \mathrm{m}$ deep cavity. The hot wire in polysilicon is suspended on this cavity and dissipates an equivalent heat of $0.5 \mathrm{~mW}$. Two contact-pads in polysilicon widen the wire towards the contacts. The total mesh number is $10^{6}$ and the total number of iterations to reach convergence is about $3 \times 10^{4}$. The mesh distribution over the hot wire is for instance $12 \times 10 \times 10$ in, respectively, the wall normal, spanwise and streamwise directions. Care has been taken to use at least 10 meshes in each direction and in each layer. Computations have been conducted in a laminar boundary layer near the trailing edge to have an equivalent 'viscous sublayer' thickness that would correspond to a fully turbulent boundary layer of $R e_{\delta}=10^{4}$, that is the Reynolds number based on the boundary layer thickness. This should be a valid comparison test case, with the wall hot film formulation developed before. What is really important, indeed, is to insure that the thermal boundary layer is small compared with the thickness of the zone wherein the velocity varies basically linearly with the wall normal distance. The streamwise length of the hot wire into the cavity in wall units is

$l_{\mathrm{f}}^{+}=\frac{l_{\mathrm{f}}}{\nu / u_{\tau}}=\frac{2(\mu \mathrm{m})}{80(\mu \mathrm{m})}=0.025$

is quite small under these circumstances. The spanwise length is consequently $l_{\mathrm{fz}}^{+}=0.6$ at this Reynolds number. It has to be pointed out that these configurations have been designed in order that the sensor be efficient in active control problems at higher Reynolds numbers encountered in real practical situations (see Tardu [1] for further discussion).

Fig. 8 shows the temperature distribution over the wall wire suspended on an air cavity. It is seen that, there is a reasonable uniform distribution of the temperature over the film indicating that the effect of the axial conduction is weakened. No secondary flows have been observed into the cavity under the hot wire during the simulations that have been conducted. This is an important point that should be confirmed by further numerical simulations, because perturbations caused by the cavity have of course to be prevented. The ratio of heat transfer from the wire to

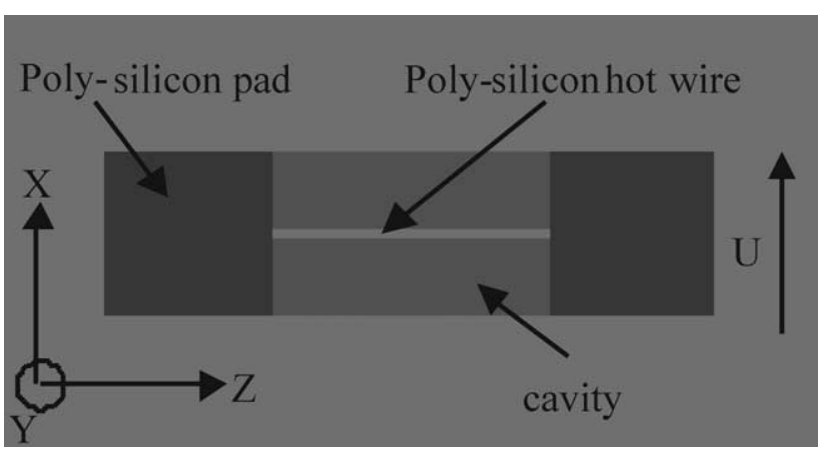

Fig. 7. Top view of the simulated hot-wire on the cavity. 

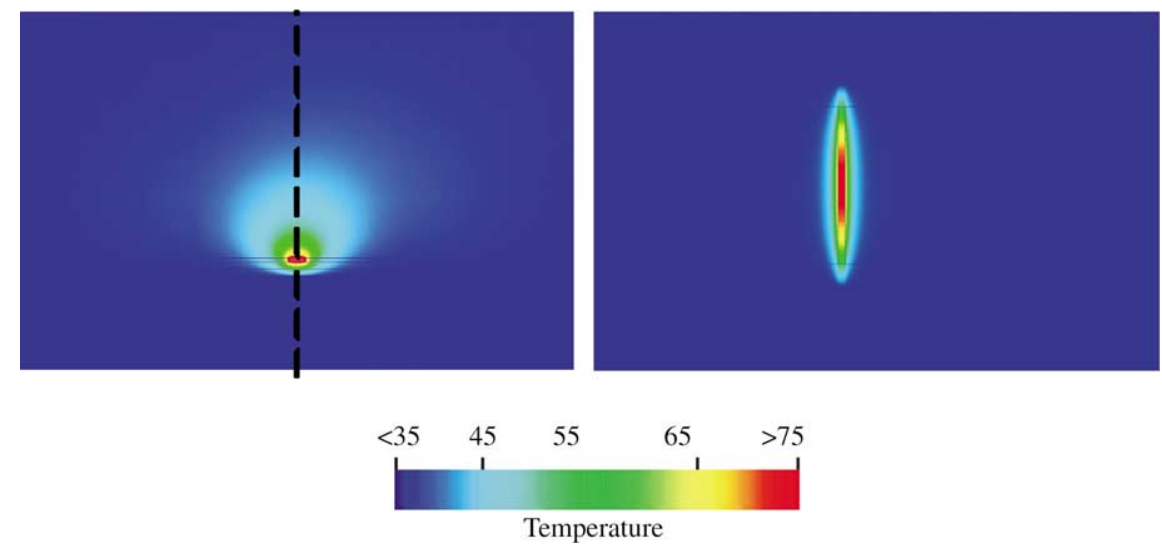

Fig. 8. Temperature distribution over the wall wire on the free micro-cavity in a transverse (left) direction together with the top view (right).

the fluid, and to the air cavity is approximately $Q_{\mathrm{fF}} / Q_{\mathrm{fS}} \approx$ 0.8 wherein we use $Q_{\mathrm{fs}}$ to express the heat flux into the cavity, since it plays the role of the substrate. This is in good agreement with the results presented in Fig. 2 concerning the heat transfer mechanism of the case water/glass for which the conductivity ratio is $k_{\mathrm{S}} / k_{\mathrm{F}}=1.27$ considering that the air cavity plays the role of substrate in which case we have $k_{\mathrm{S}} / k_{\mathrm{F}}=1.0$ Keeping the same parallelism would lead to a cut-off frequency of about $\omega^{*} \approx 0.1$ according to Fig. 2 . This cut-off frequency corresponds to

$\omega^{+}=\frac{\omega^{*}}{\operatorname{Pr} l_{\mathrm{f}}^{+2}}=200$

in wall units for $R e_{\delta}=10^{4}$ which is much more than needed, since the cut-off frequency should be $\omega^{+} \leq 10$ in wall flows. In real practical applications with $R e_{\delta}=$ $5 \times 10^{4}$, one would approximately have $\omega^{+} \approx 8$, which is still acceptable. This estimation has to be verified by dynamic computations that could not be realised with the commercial code used here.

We will investigate in more details the effect of the axial conduction by now. The axial conduction causes the nonuniformity of the temperature along the wire and is characterised by a quantity called the cold length and denoted by $l_{\mathrm{c}}$. The cold length is a characteristic distance along the wire over which the effect of the support is felt (Blackwelder [5]). The results from the simulations shown in Fig. 8 suggest that

$l^{*}=\frac{l_{\mathrm{fz}}}{2 l_{\mathrm{c}}} \approx 4$

and that the temperature is uniform along the central $50 \%$ of the wire cross-length. The current value used in thermal anemometry with platinium-iridium, or tungsten wires is about $l^{*} \approx 5$ (Blackwelder [5], Hinze [6]). As for the conduction into the substrate, the axial conduction is never zero in practical situations, yet that does not affect considerably the dynamic behaviour of the hot wire as long as the conduction losses are reasonably small. The use of polycrystalline silicon as hot wire material has a considerable advantage with respect to tungsten or platinium, because there is a factor 5-12 between the thermal conductivities. To see this, note that the ratio of forced convection to axial conduction in 1D models reads for a cylindrical hot wire of diameter $d$ :

$$
\frac{Q_{\mathrm{fC}}}{Q_{\mathrm{fF}}}=\frac{1}{2 N u} \frac{k_{\mathrm{f}}}{k_{\mathrm{F}}} \frac{1}{l_{\mathrm{fz}} / d} \frac{1}{l_{\mathrm{c}} / d} \frac{\left(T-T_{0}\right)_{\infty}}{T-T_{0}} \tanh \frac{l_{\mathrm{fz}}}{2 l_{\mathrm{c}}}
$$

where $\left(T-T_{0}\right)_{\infty}$ is the asymptotic temperature difference for a wire of infinite length, the subscripts $f$ and $F$ refer to the film (wire) and fluid as usual (Blackwelder, [5]). The thermal conductivity ratio $k_{\mathrm{f}} / k_{\mathrm{F}}$ is 2500 for platinum/air and roughly 6000 for tungsten/air, while $k_{\mathrm{f}} / k_{\mathrm{F}}$ is only 500 for polycrystalline silicon/air. Thus, to maintain the same loss ratio the MEMS sensor does not need to have $l_{\mathrm{fz}} / d$ as large as does the classical hot wires in air. The platinum wires in practice have typically $l^{*} \approx 5$ and $l_{\mathrm{fz}} / d=$ 200-500. The Nusselt number corresponding to the case investigated here is about $N u \approx 0.5$. Under these conditions the loss ratio would be as large as $Q_{\mathrm{fC}} / Q_{\mathrm{fF}} \approx 0.6$ for an equivalent conventional platinum wire of diameter $d=2.5 \mu \mathrm{m}$ and a spanwise length $l_{\mathrm{fz}}=500 \mu \mathrm{m}$ Fig. 9 shows the computational results concerning the loss ratio obtained by modifying the spanwise length $l_{\mathrm{fz}}$ and for 4

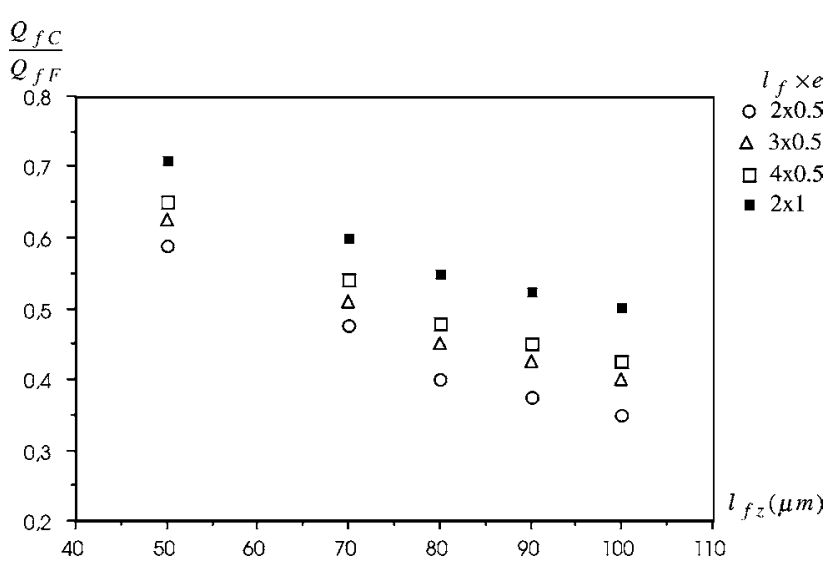

Fig. 9. The ratio of axial conduction to forced convection heat transfer for different geometries. $l_{\mathrm{f}}$ and $e$ are, respectively, the width and thickness of the wire in the cavity. The values are expressed in $\mu \mathrm{m}$. 
cases corresponding to different values of the width $l_{\mathrm{f}}$ and the thickness $e$. It is seen that even with $l_{\mathrm{fz}}=50 \mu \mathrm{m}$ one has the same loss ratio obtained with a platinum wire which is ten times longer, thus the MEMS sensor is at least as efficient as a platinum wire under these conditions.

The axial conduction decreases as $l_{\mathrm{fz}}$ increases in Fig. 9, as expected. There is a clear dispersion in this figure because of different cross-section forms. The numerical data can be organised in a different manner that also can allow the comparisons with a 1D model. One can easily show that the loss ratio for a trapezoidal wire has exactly the same form as a cylindrical wire, by using an equivalent wire diameter related to the cross-section area by (Tardu [1])

$d_{\mathrm{eq}}=\sqrt{\frac{4 l_{\mathrm{f}} e}{\pi}}$

The cold length on the other hand, varies like

$$
\frac{l_{\mathrm{c}}}{d_{\mathrm{eq}}} \propto N u^{-1 / 2}
$$

according to Blackwelder ([5]: p. 305). Using, furthermore, the classical Lévêque solution for a wall film $N u \propto \operatorname{Pr} l_{\mathrm{f}}^{+2 / 3}$ and combining leads to:

$\frac{Q_{\mathrm{fC}}}{Q_{\mathrm{fF}}} \propto \frac{1}{l_{\mathrm{fz}} / \sqrt{l_{\mathrm{f}} e}} \frac{1}{l_{\mathrm{f}}^{1 / 3} / l_{0}^{1 / 3}}$

when all other parameters are kept constant, i.e. the conductivity ratios $k_{\mathrm{f}} / k_{\mathrm{F}}, l^{*}$ and the Reynolds number. The parameter $l_{0}$ is a reference length introduced for scaling purposes. Fig. 10 shows the distribution of the logarithms of both sides. It is seen that there is an excellent collapse of the data. The slop is sensibly equal to -1 for sufficiently long wires, showing that the simple 1D model can still be applied here. The axial conduction loss is less than the one predicted by $1 \mathrm{D}$ model for shorter wires.

\subsection{Simulation of a shear stress sensor made of a polysilicon hot wire deposed on a sealed air cavity}

We have simulated other configurations dealing with a hot wire deposed on a sealed cavity. Such structures are interesting for their mechanical properties, so it is possible to obtain very long and robust hot wires without technical problems.

The model (Fig. 6d) of the wall shear stress sensor made of SOI is composed of the following layers, namely:

- a $80 \mu \mathrm{m}$-thick mono-silicon substrate

- a $1 \mu \mathrm{m}$-thick silicon dioxide film

- a $1 \mu \mathrm{m}$-thick silicon mono-silicon film

- a $1 \mu \mathrm{m}$-thick silicon dioxide film

- a $0.5 \mu \mathrm{m}$-thick polysilicon film (hot wire)

There are two possibilities with this architecture. The first one leads to a cavity obtained by etching the $1 \mu \mathrm{m}$-thick monocrystalline silicon layer situated between two silicon dioxide layers in Fig. 6d. The wire $\left(100 \times 2 \times 0.5 \mu^{3}\right)$ lies then on the oxide diaphragm as in the Liu et al.'s sensor. The computations failed to converge in this case because of huge heat transfer from the hot wire to the $\mathrm{SiO}_{2}$ membrane since the conductivity ratios of $\mathrm{SiO}_{2}$ and air is as large as 500 as we previously discussed in Section 2.2. The temperature distribution along the wire (dissipating an equivalent heat flux of $2 \mathrm{~mW}$, in order to reach a temperature of $90^{\circ} \mathrm{C}$ ) is found quite similar to that shown in Fig. 8 but the condition of continuity of heat fluxes at interfaces could not be reached. It is clear that the conduction to the $\mathrm{SiO}_{2}$ membrane affects seriously the frequency response of the probe in this situation. The second architecture allows avoiding this effect. The surface oxide film under the hot wire is released too by wet etching with hydrophilic-based solution. The hot wire lies on the air cavity through line contacts with the $\mathrm{SiO}_{2}$ membrane. The conduction is

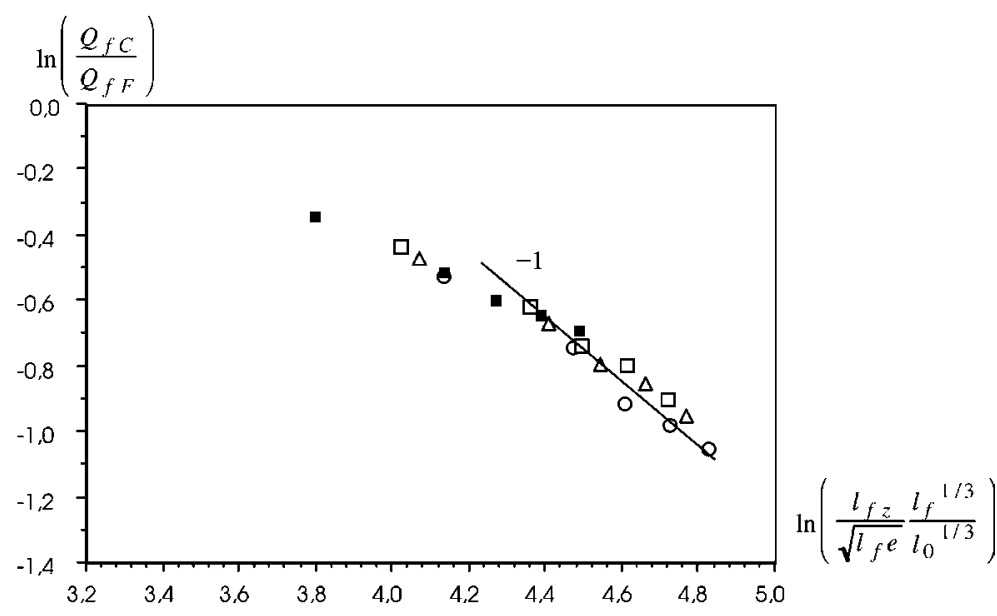

Fig. 10. Logarithm of the conductivity ratio versus the logarithm of the scaling parameter resulting from 1D model. See Fig. 9 for the symbols. 
largely avoided in this case and the results are almost identical to those discussed in Section 4.1. It has to be noted that such sealed cavity allows handling very long wires, which are more robust, decreasing the axial conduction, increasing by the way the direct convection effects. This is supposed to improve the frequency response of the sensor. It is also worth noticing that the developed technologies allow the fabrication of sealed cavities with various depths.

\section{Conclusion}

In conclusion, several architectures of hot wire wall shear stress sensor have been simulated using FVM tools. Emphasis was on the impact of the thermal insulation between the wall wire and its substrate as well as on the geometry of each layer. Free wires and wires suspended on silicon dioxide diaphragm have been investigated. An interesting technology consists of releasing both silicon dioxide layers under the wall wire and allows a good dynamic response with the advantage of being more robust. Finally, the second architecture is compatible with sensor arrays configuration and provides pressure and temperatures micro-sensors needed for a full diagnosis of the flow characteristics. Prototypes of both models are under fabrication.

\section{References}

[1] S. Tardu, L. Michelutti, Microsytèmes utilisés dans les grands écoulements Traité EGEM, Tome 6, Microfluidique, Hermès, 2003, Chapter 10 [in press].

[2] C. Liu, J.B. Huang, Z. Zhu, F. Jiang, S. Tung, Y.C. Tai, C.M. Ho, A micromachined flow shear-stress sensor based on thermal transfer principles, J. MEMS 8 (1) (1999) 90-99.

[3] P. Dengel, H.H. Fernholz, M. Hess, Skin-friction measurements in two and three dimensional highly turbulent flows with separation, in: G. Comte-Bellot, J. Mathieu (Eds.), Advances in Turbulence, Springer, Heidelberg, 1987, pp. 470-479.

[4] S. Tardu, Active control of near wall turbulence by local unsteady blowing, J. Fluid Mech. 43 (2001) 217-253.

[5] R.F. Blackwelder, Hot-wire and hot-film anemometers, in: R.J. Emrich (Ed.), Methods of Experimental Physics, Part A, vol. 18, Academic Press, New York, 1981, pp. 259-314.

[6] J.O. Hinze, Turbulence, Second ed., McGraw-Hill, New York, 1975. 